\title{
IL FEUILLETON DI BIANCA PITZORNO
}

\author{
Tanja Habrle \\ Dipartimento di studi in lingua italiana \\ Università Juraj Dobrila di Pola
}

\section{Riassunto}

Il saggio offre la visione panoramica del feuilleton nellopera di Bianca Pitzorno. Il ricordo delle opere lette da bambina, l'interesse per il mondo fugace, mutabile e complesso dell'infanzia, hanno suscitato nella Pitzorno la passione per la creazione letteraria. Pur essendo archeologa per professione, la sua vocazione verso la letteratura è stata più forte della sua passione per la ricerca. Aprendosi al mondo misterioso ed imprevedibile delle verità dei bambini, si è meritata il suo posto nella storia delle letteratura per l'infanzia con opere che spiccano per ironia e anticonformismo. Bianca Pitzorno è una delle poche autrici di letteratura infantile italiana che ha scelto delle bambine come eroine dei suoi romanzi. Essa si ispira proprio al mondo delle bambine che conosce e racconta, e di cui scrive. Le sue opere hanno per eroine le bambine che a loro volta contribuiscono a crearne altre.

Parole chiave: anticonformismo, feuilleton, ironia, letteratura per l'infanzia, protagoniste

\section{Introduzione}

Negli ultimi vent'anni si apre in Italia una nuova fase che vede scrittrici, come Bianca Pitzorno ${ }^{[1]}$, Donatella Ziliotto ${ }^{[2]}$, Beatrice Solinas Donghi ${ }^{[3]}$, impegnate a raccontare storie di bambine vivaci e ironiche.

I romanzi per l'infanzia hanno da sempre offerto a maschi e femmine fantasie diverse e spesso contrapposte, hanno suggerito sogni che sembrano prefigurare destini e modi di esistere. Per molto tempo schiacciate e devisualizzate nel racconto d'avventura, confinate negli interni o cooptate precocemente nello spazio della donna adulta, le

\footnotetext{
[1] Bianca Pitzorno (Sassari, 1942) vive e lavora a Milano. Dopo la laurea in Archeologia, ha frequentato la Scuola Superiore delle Comunicazioni. La specializzazione in cinema e televisione le ha consentito di lavorare alla RAI occupandosi di programmi sia culturali che per ragazzi. Al suo primo libro per ragazzi, uscito nel 1970, ne sono seguiti altri destinati quasi sempre a lettori pre-adolescenti. Oggi è considerata la più importante autrice italiana per l'infanzia. I suoi romanzi sono tradotti in Francia, Germania, Spagna, Polonia, Ungheria, Cile, Corea, Croazia, Grecia e Giappone. Ha ricevuto il Premio Andersen 2001 per il romanzo Tornatrás (2000). Nel 1996 l’Università di Bologna le ha conferito la laurea ad honorem.

[2] Donatella Ziliotto, nata a Trieste, è scrittrice, traduttrice, curatrice di collane, è una delle personalità più importanti delleditoria per i ragazzi in Italia. Nel 1992 ha ricevuto la nomination al Premio Internazionale Andersen per tutta la sua opera.

[3] Beatrice Solinas Donghi (Genova, 1923) oltre a essere autrice di vari romanzi, racconti e saggi è anche una delle più apprezzate scrittrici per l'infanzia. Ha ricevuto il premio Andersen 2003 con la Menzione speciale per la carriera.
} 
bambine sono state il segno di una differenza elusa. Perché si affermi un cambiamento bisogna aspettare l'entrata in scena di donne che, attraverso la scrittura, hanno rivisitato il conflitto con il mondo adulto, con la propria crescita, con la propria specificità femminile. Tra queste spiccano Louisa May Alcott ${ }^{[4]}$ e più avanti nel tempo Astrid Lindgren ${ }^{[5]}$ i cui i libri hanno segnato un vero e proprio cambiamento nella lettura rivolta alle bambine.

Emblematico il caso di Bianca Pitzorno, che è forse l'unica, nel panorama letterario italiano ${ }^{[6]}$, ad aver eletto le bambine a esclusive protagoniste dei suoi divertentissimi racconti. Le sue protagoniste sono libere e irriverenti. Viaggiano in un mondo adulto che non le convince. Sono bambine capaci di arrivare dove non è arrivata neppure Alice ${ }^{[7]}$, nel luogo dove la differenza femminile è rispettata e valorizzata.

Nellopera della Pitzorno troviamo l'esaltazione del fantastico in molte sue espressioni, un deciso distacco dal filone istruttivo e dalla favola morale. È un interessante laboratorio dove si sperimenta un incrocio di generi (giallo, fantascienza, l'avventura) e parodia, la quale trarrà il massimo divertimento proprio dal confronto e dalle continue allusioni.

Lesperienza televisiva di sette anni, dal 1970 al 1977, presso la Rai come produttrice di programmi televisivi per l'infanzia l'ha spinta ad accostamenti e incroci tra libro e altri media. La passione per il romanzo d'appendice le ha insegnato che una trama avvincente è necessaria per catturare l'attenzione. La Pitzorno è una instancabile lettrice sia del feuilleton ${ }^{[8]}$ che della letteratura dell'Ottocento e nei suoi lavori rimpasta e rimescola richiami letterari.

[4] Louisa May Alcott, scrittrice statunitense (1832 - 1888). Nel 1868 pubblicò il romanzo Little Women (Piccole donne) ispirato a ricordi autobiografici, ma eliminando la scomoda figura paterna (il noto filosofo trascedentalista Amos Bronson Alcott). Il romanzo ebbe grande successo e fu seguito da un secondo volume, nel 1869. Nel 1871 fu pubblicato Little Men (Piccoli uomini) e nel 1886 seguì Jo's Boys (I ragazzi di Jo). Con i suoi guadagni la scrittrice poté assicurare una vecchiaia tranquilla ai genitori e aiutare le sorelle.

[5] Astrid Lindgren, scrittrice svedese (1907-2002). La storia di Pippi Calzelunghe (1945) nacque per la figlia della scrittrice nel 1945. Al momento della sua pubblicazione ebbe pessima accoglienza. Lei non somigliava affatto alle protagoniste dei libri per l'infanzia di quellepoca. Astrid Lindgren, che aveva trasfuso nella sua protagonista tutta la ribellione contro l'aspetto retrivo della piccola borghesia svedese dichiarò: "Le mie bambine non fanno mai pena, se la cavano sempre, sono forti e agili, si arrampicano sugli alberi, saltano da grandi altezze proprio come i ragazzi. (...) Molte bambine degli anni ' $40 \mathrm{mi}$ scrissero, poi, da adulte, per raccontarmi quale senso di liberazione avevano provato nel leggere di Pippi, e quant'era bello che fosse una bambina e non un maschio". In F. Lazzarato e D. Ziliotto (a cura di), Bimbe, donne, bambole, Roma, Edizioni Artemide, 1987, pag. 37. I suoi libri sono tradotti in circa settanta lingue. Alla Lindgren sono stati assegnati numerosi premi tra i quali il premio Andersen Award (1963), la medaglia d’oro dell'Accademia svedese (1971) e nel 1994 le è stato assegnato il Nobel Alternativo, il premio per una vita migliore fondato nel 1980 da Jakob von Wexkull. La motivazione ricorda l'impegno di tutta una vita per i diritti dell'infanzia, la giustizia, la non violenza, la comprensione delle minoranze.

[6] Nel panorama letterario italiano rientrano autori come Bianca Pitzorno, Roberto Piumini, Beatrice Solinas Donghi, Donatella Ziliotto, Roberto Denti, Nico Orengo, Pinin Carpi che oggi ritroviamo citati fra i più rappresentativi della produzione italiana contemporanea per l'infanzia, e che pure collaborano con diversi editori, hanno iniziato a pubblicare o hanno pubblicato alcuni dei loro libri migliori proprio con la EL. Negli autori della collana è presente un nuovo modo di scrivere che rifiuta la retorica, gli stereotipi senza giustificazione (la mamma che veniva rappresentata sempre bella, dolce, laboriosa e saggia). Gli scrittori guardano con consapevole complicità alla dignità e ai diritti del ragazzo.

[7] Alice, protagonista di Alice nel paese delle Meraviglie (1865) e Alice attraverso lo specchio (1871) del reverendo Dodgson, alias Lewis Carroll. La protagonista delle due opere è una bambina, che rivendica il diritto di pensare con la sua testa. "(...) Alice offre un correttivo: quello di non lasciarsi coinvolgere da quel grande nonsense che è la vita.", in R. Denti, 100 libri scelti da R. Denti, Adriano Salani Editore, Milano, 1999, p. 63.

[8] La denominazione di 'feuilleton' inizialmente indica un tipo di pubblicazione che abbina letteratura e giornalismo. È un modulo inventato dal francese Emile de Girardin che a partire dal 1836 pubblica romanzi a puntate in appendice ai giornali, ed è rivolta al popolo che dispone così di romanzi al costo del giornale. Sono le storie di tipo consolatorio e compensatorio, imperniate sulla dicotomia buoni/ cattivi, e situate in ambientati degradati e squallidi. Maestro del genere è Octave Feuillet (1821-1890), con Il romanzo di un giovane povero. Da allora, nella storia letteraria, per indicare una certa tipologia di romanzo popolare si fa risalire il termine 'feuilleton' al nome di Feuillet, e non più alla parola 'feuillet' (che indica il foglio di giornale). Il terzo periodo, ai primi del Novecento, ha dato origine al romanzo rosa. Dal romanzo popolare deriva il modello del feuilleton, il quale a sua volta origina quella vastissima letteratura popolare che va da fine Ottocento ai giorni nostri sotto il nome di romanzo rosa. Il romanzo rosa dunque assume la struttura tipica del feuilleton, dove la coppia in antitesi è sempre quella lui/lei e dove la risoluzione finale del conflitto non si ottiene con la morte di uno dei due, bensì con la riconciliazione che origina il lieto fine. Il romanzo rosa è tuttora denominato feuilleton oppure romanzo d'appendice poiché si usava pubblicarlo a puntate in appendice a giornali e riviste, e a seconda del gradimento dei lettori veniva in seguito pubblicato in volume. In Italia la regina indiscussa del genre è Liala, pseudonimo di Amailia Liana Cambiasi Negretti Odescalchi (1897-1995), autrice di regime, che si modernizza nei termini e nelle situazioni, tuttora ristampata. 


\section{Il feuilleton di Bianca Pitzorno}

\subsection{Il romanzo}

Se la storia che scrive non lo riguarda in prima persona, se non ha radici profonde nella sua esperienza e nelle sue passioni, se non è uno specchio nel quale lui per primo possa riconoscersi e col quale dialogare, quella storia sarà asfittica, una vera minestra riscaldata e non sarà letta con vero interesse e profitto da nessuno, bambino $o$ adulto che sia. ${ }^{[9]}$

Bianca Pitzorno, la narratrice con alle spalle più di trenta romanzi per ragazzi, prima di essere una scrittrice per ragazzi è stata una ragazza lettrice, appassionata e onnivora. ${ }^{[10]}$

La Pitzorno dichiara d'essere la scrittrice per i lettori più giovani che, pur avendo pubblicato più di trenta libri, non ha mai scritto una fiaba. Non ritiene esatto definire i suoi racconti "fiabeschi" anche se contengono qualche elemento di magia: fate e vecchi alchimisti che "si comportano diversamente". La scrittrice afferma che:

Il mio modello sono, semmai, i racconti e i romanzi "filosofici" di Voltaire, dove lelemento straordinario serve soltanto per far risaltare le assurdità del nostro quotidiano. ${ }^{[1]}$

Letà dei suoi lettori varia dai sette ai tredici anni, e a tutti ha proposto dei testi che sono veri e propri romanzi.

Soltanto in una occasione ha scritto dei racconti, compresi sotto il titolo di Parlare a vanvera (1989) e La stregha di Vallebuja (2000). Neppure questi, però, si possono definire fiabe. Bianca Pitzorno è una scrittrice di romanzi. Qualcuno di taglio surreale, la maggior parte "realistici".

Bianca è una balzachiana osservante, (...) crea un nuovo cosmo, pone accanto al mondo reale un mondo proprio, con propri tipi, proprie leggi di gravitazione e un cielo proprio con proprie costellazioni: dà ad ogni personaggio, ad ogni fatto, tanto della sua stessa natura da farli diventare veri tipi. ${ }^{[12]}$

Lautrice continua le storie delle protagoniste da un libro all'altro, sulla scia della suggestione letteraria tratta da Honoré de Balzac. La stessa Pitzorno dice in apertura del libro La bambola dell'alchimista (1988) che da Balzac ha appreso "l'arte" di far circolare i personaggi da un libro all'altro.

Nei suoi romanzi si intrecciano l'avventura, la fantasia, la storia ed uno spiccato umorismo. Perciò i suoi sono romanzi d’avventura, di fantasia, di formazione e di storia.

I racconti scritti per i più piccoli si ispirano a «storie del quotidiano», della vita di tutti i giorni, dove la meraviglia nasce proprio dall'irrompere dell'imprevisto nelle abitudini banali e scontate. Le storie per i più grandi possono rientrare nella categoria dell'avventura. In questa categoria rientrano sia i romanzi propriamente storici ( $\mathrm{La}$

[9] Lazzarato, F., (a cura di), Scrivere per bambini, introduzione di Bianca Pitzorno, Mondadori, Milano, 1997, p. 23.

[10] Tra le protagoniste dei libri per l'infanzia che hanno inciso maggiormente sulla formazione di Bianca Pitzorno è Marigold di Lucy M. Montgomery. L'opera descrive un percorso di crescita carico di profonde risonanze psicologiche, e la serie di Bibi di Karin Michaelis, sconvolgente scoperta di messaggi d'avventura e di libertà per le ragazze del primo dopoguerra.

[11] Pitzorno, B., Storia delle mie storie, Nuova Pratiche Editrice, Parma, 1995, p. 48.

[12] Faeti, A., I diamanti in cantina, Bompiani, Milano,1995, pp. 38 e 40. 
bambina col falcone, Con la carovana di Alessandro) sia gli altri (Speciale Violante, Principessa Lauretina, Polissena del Porcello, Diana, Cupído e il Commendatore).

Lambito storico, che Bianca Pitzorno frequenta assiduamente con rivisitazioni originali, le suggerisce nuovi stimoli di riflessione sullinesplorato mondo femminile, mentre al fiabesco attinge inserendo forti venature parodistiche. Nascono così storie di piccole donne intrepide e beffarde che si scontrano, mai accomodanti, con il mondo adulto, attraversano una trama narrativa sorretta da tutte le risorse della fiaba e dell'avventura, spesso riscritte e capovolte con straordinaria inventiva.

Centrale nei suoi romanzi è l'invenzione di personaggi che poi vivono una vita autonoma, la creazione di mondi paralleli capaci di aprire un nuovo rapporto tra la realtà e la sua possibilità.

Lappartenenza sessuale si presenta immediata in lei che, toccata sia pure indirettamente dalla cultura del femminismo, è convinta che si possa scrivere solo di quello che si conosce all'interno ${ }^{[13]}$.

Unaltra caratteristica che colpisce nella produzione di Bianca Pitzorno sono le sue protagoniste, "forti lettrici". Parlano fra loro di libri, se li presentano, ne vengono influenzate. Questo ha spinto alcune lettrici ad affrontare libri per adulti e a scrivere, alla Pitzorno, per ringraziarla di aver letto, su "indicazione" dei suoi personaggi, diverse opere: dai gialli di Aghata Christie a Il Signore degli anelli di John Ronald Reuel Tolkien.

\subsection{Ascolta il mio cuore}

Nel libro di Bianca Pitzorno Ascolta il mio cuore (1991), che esce riproponendo la collana "Contemporanea" della Mondadori e si avvale delle immagini di Quentin Blake ${ }^{[14]}$, ci sono atmosfere lontane e sensazioni rivolte verso il futuro. Il volume contiene molte tematiche e si pone di fronte a esse in termini quasi riassuntivi, perché nelle sue pagine c’è, molto viva e articolata, l'intera visione dell'infanzia a cui l'autrice ha dedicato tanti libri.

Lambito in cui siamo immersi, dall'inizio alla fine, è quello del conflitto ${ }^{[15]}$. Le tre protagoniste dell'opera sono fiere, astute, mai sfiorate dai turbamenti di un pacifismo

[13] Bianca Pitzorno, infatti, si ispira a bambine che conosce e per le quali comincia a raccontare. Le ragazze si sentono valorizzate da questa attenzione e possono rispecchiarsi quali protagoniste di racconti in cui si riconoscono e che contribuiscono a creare. La caratteristica dei romanzi di Bianca Pitzorno è il rapporto di complicità femminile, ispirato alla libertà e al rispetto, che inserisce specifiche modalità di relazione tra donna e ragazza fondate su uno sguardo valorizzante, attraverso scambi di pensieri, incoraggiamenti, scoperte. Esistono persone grandi capaci di prendere in considerazione una possibile autonomia infantile e di dar giusta importanza ai problemi che le ragazze ritengono vitali. Di solito ce nè sempre uno, di sesso femminile, connotato da amabile stravaganza: la fata di Lavinia, la mamma speleologa di Clorofilla, l'amica della piccola Aglaia in La casa sullalbero.

[14] Quentin Blake (Inghilterra, 1932) è disegnatore, illustratore e scrittore. Vive e lavora tra Inghilterra, Italia e Francia. È uno degli illustratori contemporanei più famosi al mondo. Ha ottenuto un successo internazionale illustrando i racconti e i romanzi di Roald Dahl. Nel 2002 ha vinto la Hans Christian Andersen Medal per il suo contributo alla letteratura per l'infanzia. Nel 2007 è stato nominato Officier des Artes et des Lettres.

[15] Elisa, Prisca e Rosalba sono tre alunne di una quarta elementare femminile nella Sassari dell'autunno del 1949 e, mentre comincia la scuola, scoprono di avere una nuova maestra, che si chiama Argia Sforza, ribattezzata opportunamente Arpia Sferza. La maestra ha le mani viscide, è crudele, ipocrita e untuosa. Il libro è scandito dai mesi che compongono l'anno scolastico e viene ritmato dalle fasi di una battaglia, via via sempre più esplicita e dura, combattuta dalle tre amiche e dall'insegnante. Il conflitto è sempre più aperto e crudo. Elisa, Prisca e Rosalba sono espressioni molto attraenti di un vitalismo gioioso, incontenibile, ma insieme partecipe dei dolori del mondo. Come in tutti i momenti in cui il processo educativo deve fare i conti con le fasi cruciali da cui è definito, anche in questo anno di battaglie, è soprattutto in gioco la credibilità dell'insegnante, quindi, quella di tutti gli adulti. La signora Sforza è corrotta, interamente, da una società che si basa soprattutto sullopera di persone come lei: umilia, bastona, espelle, deride le allieve povere e si inchina a quelle ricche. Procede in quell'itinerario educativo che è il luogo dellesclusione, perché trasforma in domestiche, cacciandole dalla scuola, le due sole scolare che davvero non vuole conservare insieme alle altre. La maestra Arpia guida la sua classe con la viltà. 
arrendevole e accomodante, sempre decise a guardare diritto in faccia il nemico. Contro la maestra non si perdono in burle, cercano solo di colpirla duramente e direttamente in quella che hanno scoperto essere la sua essenza. La maestra Sforza ha una vera e propria sindrome, si piega verso i potenti, verso i danarosi con un incredibile godimento da schiava soddisfatta. Così la strategia complessiva delle tre congiurate è rivolta a demolire le occasioni in cui la signora Sforza può meglio evidenziare la sua vocazione servile.

La trama del libro non riguarda solo la durissima guerra mossa da tre alunne a una maestra classista. Ascolta il mio cuore è anche un romanzo di formazione con venature parodistiche là dove Prisca scrive sul diario i suoi «racconti mensili» trasformando in 〈feuilleton〉 la vita di tutti i giorni.

Prisca scrive lunghi temi che ci vengono integralmente offerti in lettura. Così, accanto alla cronaca dellanno scolastico, abbiamo anche queste pause di riflessione, in cui seguiamo la fantasticheria infantile, con grande varietà di toni e di argomenti. Bianca Pitzorno ha voluto conservare le tracce meno appariscenti di una storia in gran parte davvero vissuta. Il libro è pieno di giocattoli attentamente datati, di cibi, di vestiti, di libri, di atteggiamenti, di costumi. Prima della narrazione cè una «presentazione», che risponde bene al suo compito, quello di spiegare ai giovanissimi in che cosa consistono le vere differenze tra le epoche. La "presentazione» è un catalogo di oggetti scomparsi, di comportamenti obliati, di consuetudini che sembrano eterne.

\section{A proposito del libro Bianca Pitzorno scrive:}

Ci misi vent'anni a decidermi, (...) per me fu un viaggio all'indietro molto doloroso. Al di là degli aneddoti, tornai a soffrire sulla carne viva gli stessi sentimenti, la stessa rabbia impotente, la stessa disillusione riguardo all'aiuto che un bambino che subisce l'ingiustizia può aspettarsi dagli adulti. E cercando di mettere in scena, di rappresentare quell'antica tragedia, non mi ritrovai con un personaggio "vecchio e raggrinzito", ma con una ferita sempre giovane, viva e sanguinante (...) Ogni tanto andavo a parlare con Marisa, che era stata mia compagna di scuola fin dalla prima elementare e con me aveva condiviso quell'esperienza. Ci rispolveravamo a vicenda i ricordi (...) Nel romanzo elaborai tutto questo materiale, lo integrai con altri episodi avvenuti nella scuola, ma in altre classi. Inserii gli episodi in una struttura scandita dai componimenti della protagonista Prisca Puntoni, che non erano i miei veri temi di allora, tutti perduti, ma somigliavano in modo plausibile a quello che scrivevo davvero. Scomposi e ricomposi le caratteristiche fisiche e psicologiche di molte compagne per creare i personaggi delle tre protagoniste. Insomma, passai il tutto al filtro della finzione letteraria. Ma il tempo, i problemi, il clima restava quello dei miei personali anni cinquanta. ${ }^{[16]}$

È lecito pensare che, scrivendo il libro, la Pitzorno abbia voluto verificare il proprio assorbimento dei fatti, esplorare l'ampiezza e la tenuta dei propri ricordi. Ed eccolo qui, dunque, l' "ingrediente Balzac" cui accennava Antonio Faeti ${ }^{[17]}$, che si può riconoscere anche nel libro Polissena del Porcello.

Una delle chiavi del libro sta anche nel titolo. Così, Ascolta il mio cuore è solo la frase con cui Prisca si rivolge alle amiche quando sente scoppiare dentro di sé una

[16] Pitzorno, B., Storia delle mie storie, op. cit., p. 116.

[17] Antonio Faeti (Bologna, 1939) è docente di Grammatiche della fantasia presso l'Accademia di Belle Arti di Bologna, e ha scritto numerosi e importanti saggi sulla letteratura per l'infanzia, l'illustrazione, il fumetto e la comunicazione di massa. Bianca Pitzorno ritiene il romanzo dell'Ottocento fondamentale per la propria formazione. Lautrice continua le storie delle protagoniste da un libro all'altro, sulla scia della suggestione letteraria tratta da Balzac. La stessa Pitzorno dice in apertura del libro La bambola dellalchimista che da Balzac ha appreso "larte" di far circolare i personaggi da un libro allaltro. "Sono indispensabili: una ricchezza immensa di fatti inspiegabilmente assorbiti; uno sguardo avido; una sete da vampiro; talento da eccelso magazziniere connesso con un deposito-memoria stupendamente attrezzato per la conservazione". In A. Faeti, I diamanti in cantina, op. cit., 1995, p. 43. 
specie di bomba a causa delle ingiustizie a cui assiste e, pertanto, chiede che le misurino i battiti, posando una mano sul suo petto. Il titolo si rivolge anche a un'infanzia messa in subbuglio dai primi innamoramenti. Prisca, infatti, si innamora del dottor Maffei, per attirare l'attenzione del quale chiede di essere auscultata.

Innamorarsi a sette anni e mezzo non è facile, ma Prisca sapeva come fare, perché lo aveva visto tante volte al cinema e sui fotoromanzi di Ines. ${ }^{[18]}$

Lautrice ci ricorda che siamo, tra l'altro, nell'epoca del grande fenomeno popolare prodotto dal fotoromanzo.

Cè una storia d'amore che vede tra i protagonisti le tre bambine, il maturo cardiologo e un'affascinante professoressa di matematica alla quale viene dedicato un vero e proprio culto, con tanto di composizioni letterarie in suo onore. Però il gioco del titolo non finisce qui. Esso consente di delineare un itinerario di letture che non permette di escludere il Cuore di Edmondo De Amicis, spesso chiamato in causa da chi non può evitare di rammentare quell'altra cronaca di un anno scolastico, mentre legge questa, piena di tumulti e risse, ma scandita allo stesso modo, secondo un tempo mitico che è solo scolastico. Il libro della Pitzorno:

(...) stravolge quella particolare modalità di approccio all'infanzia contenuta in De Amicis. L'Enrico di Cuore, infatti, non rivela mai sentimenti suoi, è - in un certo senso - filtro dei bisogni e delle aspettative del mondo adulto. Ascolta il mio cuore, invece, entra in quelle emozioni che pervadono il mondo dell'infanzia a prescindere da ciò che i grandi pensano e dicono. ${ }^{[19]}$

Ascolta il mio cuore è anche un interessante libro sull'amicizia femminile ${ }^{[20]}$, tema importante e ricorrente nella produzione della Pitzorno. Lo troveremo nei suoi romanzi: Speciale Violante (1989), Principessa Lauretina (1991) e Polissena del porcello (1993).

\subsection{Diana, Cupído e il Commendatore}

Il romanzo Diana, Cupído e il Commendatore (1994) è romanzo d'avventura, di formazione e umoristico, e si avvale delle illustrazioni di Quentin Blake. Nellopera Bianca Pitzorno riporta la narrazione quasi dove si era concluso Ascolta il mio cuore, ma l'angolo di visuale è diverso. ${ }^{[21]}$

Diana, Cupido e il Commendatore è ambientato negli anni cinquanta. Nel romanzo l'autrice affronta di nuovo i giorni della sua infanzia, il clima di quegli anni colmi di censure, di divieti, di ammiccamenti e sviluppa a modo suo un tema di formazione, di

[18] Pitzorno, B., Ascolta il mio cuore, Mondadori, Milano, 1991, p. 38.

[19] Pontremoli, G., Come cammina la metà del cielo, intervista a B. Pitzorno, in "École", n. 5, 1992, p. 33.

[20] Le protagoniste imparano, durante un anno di scuola, che nella vita davanti all'ingiustizia bisogna lottare senza arrendersi mai: fiere e astute non perdono occasione per demolire il servilismo della signora Sforza. E la solidarietà, l'ostinazione, l'allegria, la fiducia per i grandi ideali danno alle eroine una forza tutta particolare. Bisogna ancora dire che Prisca Puntoni, in questo libro come in Diana, Cupído e il Commendatore non è il ritratto di Bianca Pitzorno da piccola. A Prisca lei somigliava soltanto per la precoce vocazione alla scrittura. [21] Ora la protagonista è Diana, dodicenne, che deve cambiare città perché il patrigno, il bel Manfredi, ha venduto tutti i non pochi beni della madre. Così non hanno più nulla e, con la meravigliosa sorellina Zelia, che è in seconda elementare, devono bussare alla porta del nonno. Il Commendator Serra è un uomo "fatto da sé", ricchissimo, settantaduenne burbero, rozzo, arrogante, scostante e possiede tutte le sale cinematografiche della città. Da sempre è in lite con la madre di Diana perché lei è snob, bella, inconsistente, attratta solo dalla superficie e non dalla sostanza. 
educazione sessuale, istruttivo, con l'occhio rivolto al presente. Per questo l'opera risulta molto leggibile e utile.

Nell'introduzione la Pitzorno scrive:

Tanto per cominciare non c'era ancora la televisione. E non c'era quindi, fra le altre cose, la pubblicità martellante degli assorbenti igienici che oggi non permette a nessuno, neppure a un bambino di tre anni, di ignorare che le ragazze e le donne, ogni mese, hanno le mestruazioni. ${ }^{[22]}$

Negli anni in cui Diana era adolescente tale ignoranza era più che consentita, anche a lei e alle sue coetanee.

A loro due non sembrava di essere molto cambiate dall'anno prima, a parte il fatto di essere cresciute in altezza di qualche centimetro e, per quanto riguarda Diana, di aver messo gli occhiali. Per fortuna né all'una né all'altra era ancora cresciuto il petto, né gli era successa quella cosa misteriosa che tutti nominavano sottovoce e con mille nomi diversi, nomi assurdi come rosso di sera, oppure il marchese, o ancora le mie cose (mie di chi?), e il cui risultato era diventare signorina (ma che cosa voleva dire esattamente? Gaia Antenori le domestiche la chiamavano signorina da quando aveva tre anni, e la governante inglese miss). ${ }^{[23]}$

In questo romanzo incontriamo le parole "necessarie", alle quali non si può né si deve rinunciare. Perché, sempre nello stesso romanzo, l'autrice racconta del disagio della ragazzina cui uno sconosciuto, al cinema, tocca una gamba, spostando la mano lentamente dal gionocchio alla coscia. È un episodio scabroso ma frequentissimo. Come osserva Faeti, la Pitzorno ci racconta:

(...) la storia dell'Eros, come storia culturale, storia dei costumi, storia delle mentalità collettive. ${ }^{[24]}$

Cupido, il Cupido dipinto in una camera di questa strana villa neogotica dove il ricco e burbero Commendatore ha accolto le tre derubate, davvero stuzzica, sovrintende a molte delle tante vicende descritte in queste pagine. Il piccolo arciere alato colpiva all'impazzata, coinvolgendo nel suo gioco persone d’ogni età e di ogni condizione sociale, ricchi e poveri, eroi greci e infermieri del manicomio, zii, suore e guerrieri pellerossa, cameriere e cantanti lirici, scimmie di pezza e compagni di banco secchioni. Sembrava che l'amore fosse l'unica forza a governare e a mettere in subbuglio il mondo.

Il nonno Serra era forte e aggressivo e contrapposto agli stereotipi libreschi di Diana, che aveva sempre associato al concetto di vecchiaia un'idea di grande fragilità e tenerezza, ma che deve prendere coscienza che non è sempre così. Ha preparato, inconsapevolmente, se stessa alla battaglia che costituirà lo sviluppo dell'intreccio: il nonno Serra, con tutta la sua forza, cede a Cupido e a una sarta teatrale, ma, quando decide di sposarla, un complotto di famiglia lo conduce dritto dritto in manicomio, da dove lo salveranno Diana e Zelia. Ma potranno liberarlo proprio perché Diana conosce, valorizza e apprezza la taurinità senile.

Tra le pagine del romanzo l'autrice inserisce la storia dell'Iliade, così come è recepita da una ragazzina che ne affronta la lettura in seconda media e così come la racconta, in forma epistolare, a un'amica che ancora non la conosce.

[22] Pitzorno, B., Diana, Cupído e il Commendatore, Mondadori, Milano, 1994, p. 5.

[23] Ivi, p. 18.

[24] Faeti, A., I diamanti in cantina, op. cit., p. 144. 
Cara Teresa,... questa è la storia originale, ed è scritta in modo davvero appassionante. Ci sono dei punti che ti fanno venire i brividi, come quando il dio del sole Febo Apollo (fratello gemello di Diana) scaglia le frecce con l'arco d'argento per spargere un'epidemia nell'accampamento dei greci. Elisa Maffei, che ha uno zio medico, dice che dovrebbe essere colera, oppure peste bubbonica. Fatto sta che i guerrieri cominciano a morire come le mosche e gli amici devono bruciare i loro cadaveri su grandi roghi. E tutto per colpa di Agamennone che aveva trattato male un certo Crise, sacerdote di Apollo. ${ }^{[25]}$

Il racconto epistolare prosegue e illustra non solo gli elementi della trama, ma il modo in cui personaggi e vicende vengono trasportati nel quotidiano e diventano oggetto di accese discussioni e sfide tra i compagni di classe. Rita Valentino Merletti ${ }^{[26]}$ nota che

Il modo di raccontare di Bianca Pitzorno, (...) il tono colloquiale, spiccio, dettato dall'urgenza del narrare lascia trasparire un fortissimo desiderio di condividere qualcosa di appassionante e coinvolgente. ${ }^{[27]}$

Perché i libri e la vita reale, come scoprirà Diana alla fine della sua avventura, spesso si assomigliano, ma non sono mai la stessa cosa.

\subsection{Polissena del Porcello}

Polissena del Porcello è un altro interessante libro sulla ricerca della propria identità, delle proprie origini e sullamicizia femminile. Qui l'amicizia tra la protagonista e Lucrezia, entrambe alla ricerca delle proprie origini, conosce prove difficili e situazioni limite che conducono addirittura a rischiare la vita nelle segrete di un castello reale. Ma anche complicità nel gioco di travestimenti e nello scambio di ruoli, in una trama tra il feuilleton e il giallo, tra la finzione e la verità della "condizione bambina". Il romanzo è ricco di citazioni, nascosti riferimenti letterari, improvvisi scarti narrativi e colpi di scena.

Anche questa volta la Pitzorno è andata a scavare fra sentimenti ed emozioni che sembrano senza tempo. Ha rispolverato l'antico ed eterno dubbio che assale tutti i bambini: "Sarò davvero figlio di questi genitori?" e ha ambientato Polissena del Porcello in un passato non molto ben definito, che può essere il Cinquecento come il Settecento. Il romanzo narra le avventure di Polissena vissuta felicemente fino all'età di undici anni nella sua casa di Cepaluna, circondata dall'affetto dei suoi cari. Ma un bel giorno scopre per caso la tremenda realtà che tutti le hanno sempre nascosto: è una trovatella, una figlia adottiva. La rivelazione fattale la sconvolge. Perciò scappa di casa alla ricerca dei suoi veri genitori. E fila diritto verso il convento delle suore di clausura, dove presume di essere stata abbandonata. E qui assistiamo alla "scena della Ruota" che è il topos fondante del feuilleton:

Senza accorgersi d'esser visto, il giovanotto si avvicinò alla ruota, che si apriva nel muro del convento, di fianco al portone, e fece ruotare il cilindro di legno in modo da farne apparire allesterno la cavità. "Sto assistendo allabbandono di un neonato?" si chiese Polissena, col cuore che le martellava forte nel petto. Che

[25] Pitzorno, B., Diana, Cupído e il Commendatore, op. cit., p. 92.

[26] Rita Valentino Merletti, studiosa di letteratura per l'infanzia, ha perfezionato la sua preparazione negli Stati Uniti, studiando e lavorando presso il Simmons College e la Boston University.

[27] Merletti, R. V., Raccontar storie, Mondadori, Milano, 1998, p. 64. 
fosse un segno del destino? Il giovane depose con precauzione il suo fardello sulla base del cilindro, poi con un colpetto lo fece ruotare, e quello trasportò un suo contenuto all'interno delle grosse mura. Si udì uno strillo altissimo. ${ }^{[28]}$

Ma chi strilla, qui, non è un neonato che per sua sventura viene recato alla Ruota da un padre snaturato o da un suo incaricato, bensì un porcello di latte che un onesto giovanotto porta in regalo alla Madre Superiora per ringraziarla dei buoni uffici da lei offerti per una rappacificazione con la sua fanciulla del cuore.

I segreti del convento fanno emergere alcuni oggetti che si rivelano altrettanti indizi sulle sue origini: uno scrigno che contiene un pesciolino di corallo, una sciarpa a righe, un brandello di bandiera pirata, una calza maschile di seta rossa. In questa sua difficile ricerca trova Lucrezia, una coraggiosa bambina girovaga che l'accoglie in seno alla sua compagnia di animali acrobatici. Gli indizi sono complessi e il viaggio procede, di sorpresa in sorpresa, fra colpi di scena che ribaltano in modo del tutto inatteso la situazione.

La ragazzina, girando con la sua amica Lucrezia trova varie persone che ogni volta sembrano essere i suoi genitori. Di famiglia in famiglia, di luogo in luogo, lei, Lucrezia e la Compagnia di Animali Acrobatici giungono a una specie di locanda sadiana (la Civetta Verde), abitata da nobili dissoluti e violenti e da servette e sguattere al loro servizio. Lì tengono uno spettacolo e poi via, fino a che arrivano a corte.

Polissena è convinta di essere una principessa e invece viene arrestata per lesa maestà. In prigione, ecco apparire un uomo da un buco nella cella, proprio come l'abate Faria. Ma non è Faria, bensì un pediatra reale, l'unica persona che può rivelare che Polissena è una trovatella. Al tempo del suo ritrovamento era avvolta in una camicia con delle misteriose iniziali. Seguendo le tracce della misteriosa camicia, Polissena e Lucrezia incontrano uno stilita (proprio come Simon del deserto di Buñuel) e gli raccontano tutta la storia della trovatella e della camicia. Lo stilita prima cade in catalessi e poi confessa la verità. Si scopre così che la madre adottiva di Polissena era un tempo fidanzata con l'eremita, il quale rapì la bambina per gelosia. Più tardi, la bambina, come trovatella, venne adottata... dai suoi stessi genitori. E infine, arriva un altro colpo di scena: l'acrobata Lucrezia è la vera principessa, prima creduta morta, diventata vagabonda e poi, tornata a corte, di nuovo e definitivamente regina.

Incontri, riconoscimenti, intrighi, vendette, confessioni di antichi delitti, equivoci e incredibili coincidenze portano sempre Polissena a un passo dalla verità. Ma ogni volta lei ricomincia da capo. La soluzione del mistero sarà proprio quella che nessuno si aspettava. Di chi sarà figlia Polissena, bambina borghese un po' snob, col sogno di essere figlia di un principe? Del povero pescatore di corallo con una pesante famiglia alle spalle, dell'elegante e crudele pirata morto impiccato, dell'anziana e simpatica contadina arricchita, dell'ipocrita locandiera $\mathrm{o}$, invece, del re?

La storia si conclude con la punizione dei cattivi, con la trasformazione della locanda sadiana in una scuola e con il gran rifiuto di Lucrezia, che decide di soprassedere ai suoi doveri di regina e di fare la saltinbanca. Polissena invece torna a casa Gentileschi, il suo nido di Cepaluna da cui mai più si allontanerà.

[28] Pitzorno, B., Polissena del Porcello, Mondadori, Milano, 1993, pp. 16-17. 
Ci sono tre elementi principali in Polissena del Porcello: la paura di non essere figli dei propri genitori, il mito della principessa, e la potenza e la ricchezza associate alla crudeltà, i potenti sono corrotti, in questo senso, i borghesi benestanti rappresentano l'equilibrio, la saggezza. Loro sono le brave persone del romanzo.

Nei romanzi della Pitzorno traspare evidente il gusto di prendere dei personaggi immersi in un ambiente tranquillo, protettivo e poi di colpo farli precipitare in una situazione di grande insicurezza materiale e spirituale. Questa improvvisa messa a nudo fa affiorare le qualità migliori, magari nascoste, dell'eroina di turno.

La forza di Polissena sta nella volontà positiva di reagire che, senza volerlo, diventa storia e fa la storia. Polissena è un tipo particolare, un po' snob, molto determinata a conoscere le sue vere origini e speranzosa di scoprirsi autentica principessa.

Ci sono in tutto il romanzo molti omaggi letterari, molti luoghi comuni del feuilleton, e tanti riferimenti cinematografici.

(...) Certamente Senza famiglia poi De Sade, coi suoi castelli pieni di nobili violenti e dissoluti, naturalmente Il conte di Montecristo e soprattutto Il principe e il povero, con i suoi scambi di ruoli che colpiscono profondamente la fantasia di bambine e bambini. Poi Buñuel, e infine un gusto diffuso in tutto il libro per la parodia, anche nei momenti più drammatici. ${ }^{[29]}$

Lautrice ci conduce in un gioco narrativo dove la letteratura è rifacimento, maliziosa rivisitazione, poetica del remake, piacere del riconoscimento di topoi già noti e colta rilettura. È in questa brillante mappa di citazioni e di rinvii l'occasione per offrire alle ragazze messaggi di libertà. Tra questi lo smontaggio ironico di un mito, certamente alimentato dai mass-media: quello della principessa (le vicende della principessa Diana d'Inghilterra in questo senso sono state estremamente amplificate dalla stampa). Uno degli aspetti parodistici del libro, è che essere una principessa non è per niente una cosa bella, perché la principessa appare cattiva o infelice, prigioniera dell'etichetta, delle regole e della ragion di stato. E il feuilleton si è rivelato il mezzo più adatto per affrontare con umorismo questo tema.

L'illustratore inglese Quentin Blake, abilissimo nel disegnare animali con un grado di antropomorfizzazione misurato ed efficace, offre al libro il suo stile "acrobatico", perfettamente in sintonia con la vicenda.

La giovane Gentileschi è un altro delizioso ritratto di ragazza intelligente e moderna, l'altra faccia delle protagoniste incontrate in Speciale Violante, Principessa Lauretina e in Ascolta il mio cuore.

[29] Caviezel, G., Polissena del Porcello. Ovvero bambine in cerca di radici nell'ultimo romanzo di Bianca Pitzorno, in "Sfoglialibro" n. 4, 1993 , p. 7. 


\section{CONCLUSIONE}

Bianca Pitzorno è una scrittrice che al pubblico dei più giovani ha dedicato da trentacinque anni tutto il suo lavoro e che ha conquistato al piacere del testo moltissimi lettori.

Il laboratorio della Pitzorno sperimenta un incrocio di generi, dal fantastico all'umorismo, dal 'feuilleton' allavventura, ed è sciolto dalle esigenze pedagogiche tradizionali e dalla moda. La sua lingua è sapiente e briosa, il risultato di grande cultura e consapevolezza.

Le protagoniste delle opere della Pitzorno fanno parte della narrativa contemporanea e sono inserite in trame che sfruttano i grandi archetipi del passato: il mistero da risolvere, le complicazioni familiari, il rapporto con gli adulti, il viaggio avventuroso. Le grandi categorie dell'avventura, della solitudine, del coraggio premiato, del dolore, del lieto fine sono sempre attuali, rivissute, comè naturale, in modo più disinvolto e soprattutto con una grande autonomia delle protagoniste. Le sue protagoniste sono dotate di colto umorismo, si muovono spigliatamente nell'avventura, anche quella del quotidiano, senza dimenticare quella cultura fiabesca che ha sempre offerto alle ragazze unaltra capacità di conoscere il mondo, di riviverlo e di modificarlo.

\section{BIBLIOGRAFIA}

AA.VV., 1979, Enciclopedia Garzanti della letteratura, Aldo Garzanti Editore, Milano.

BESEGHI, E. (a cura di), 1994, Nel giardino di Gaia, Mondadori, Milano.

BITI, V., 1997, Pojmovnik suvremene književne teorije, Matica Hrvatska, Zagreb. BOERO, P., DE LUCA, C., 1996, La letteratura per l'infanzia, Laterza \& Figli Spa, Roma-Bari.

BOERO, P., 1997, Alla frontiera, Edizioni EL, Trieste.

CAVIEZEL, G., 1993, Polissena del Porcello. Ovvero bambine in cerca di radici nell'ultimo romanzo di Bianca Pitzorno, in Sfoglialibro, n. 4, 2-7.

DENTI, R., 1999, 100 libri scelti da R. Denti, Adriano Salani Editore, Milano.

DUDA, D., 2002, Kulturalni studiji, Ishodišta i problemi, AGM, Zagreb.

FAETI, A., 1995, I diamanti in cantina, Bompiani, Milano.

HABRLE, T., 2004, Personaggi femminili di Bianca Pitzorno, KIT GRM, Pula.

LAZZARATO, F. (a cura di), 1997, Scrivere per bambini, introduzione di Bianca Pitzorno, Mondadori, Milano.

LAZZARATO, F., ZILIOTTO, D., (a cura di), 1987, Donne, bimbe, bambole, Dartemide, Roma.

LURIE, A., 1999, Non ditelo ai grandi, Mondadori, Milano.

MERLETTI, R., V., 1998, Raccontar storie, Mondadori, Milano.

NÖTH, W., 2004, Priručnik semiotike, Ceres, Zagreb.

PITZORNO, B., 1989, Speciale Violante, Monadori, Milano.

PITZORNO, B., 1991, Principessa Laurentina, Mondadori, Milano.

PITZORNO, B., 1991, Ascolta il mio cuore, Mondadori, Milano. 
PITZORNO, B., 1993, Polisséna del Porcello, Mondadori, Milano.

PITZORNO, B., 1994, Diana, Cupido e il Commendatore, Mondadori, Milano.

PITZORNO, B., 1995, Storia delle mie storie, Nuova Pratiche Editrice, Parma.

PITZORNO, B., 1987, Reale come la vita, bello come unavventura, in Riforma della scuola, n. 8 e 9, 70-76.

PONTREMOLI, G. 1992, Come cammina la metà del cielo, intervista a B. Pitzorno, in École, n. 5, p.33.

SOSSI, L., 1998, Metafore d'infanzia, Edizioni EL, Trieste.

\section{FELJTON U KNJIŽEVNOM DJELU BIANKE PITZORNO}

Predmet rada je panoramski prikaz primjera feljtona kroz djela Bianke Pitzorno.

Sjećanje na pročitana djela iz djetinjstva, zanimanje za prolazan, nestalan i složen svijet djeteta bili su prepotentan zov književnici za pisanje. Inače arheologinja po zanimanju, jednostavno je preselila u svoju književnu vokaciju strast za istraživanjem. Otvorivši se tajanstvenom i nepredvidljivom svijetu dječjih istina, Bianca Pitzorno se smjestila u povijest dječje književnosti s knjigama koje se ističu po ironiji i antikonformizmu. Bianca Pitzorno možda je jedina u talijanskoj književnoj panorami koja je izabrala djevojčice kao jedinstvene i glavne junakinje svojih zabavnih romana. Naime, ona se nadahnjuje djevojčicama koje poznaje i o kojima, uostalom, pripovijeda, a zatim piše. Djevojčice se prepoznaju kao glavne junakinje te sudjeluju pri stvaranju novih.

Ključne riječi: antikonformizam, feljton, dječja književnost, junakinje

\section{FEUILLETON IN THE LITERARY WORK OF BIANCA PITZORNO}

The essay offers a panoramic view of the feuilleton in the literary work of Bianca Pitzorno.

The memory of the works read as a child, the interest in the fleeting world, mutable and composed of children, have aroused Pitzorno's passion for literary creation.

Despite being an archaeologist by profession, her vocation for literature has been stronger than her passion for research. By opening herself to the unpredictable world of mystery and truth of the children, she has earned her place in the history of children's literature with works that stand out for humor and unconventionality. Bianca Pitzorno is one of the few Italian authors of children's literature who has chosen the girls as heroines of her novels. She comes out right in their world in what she knows, tells and writes. Her heroines, in her works, contribute in turn to create new ones.

Key words: nonconformity, feuillton, children's literature, protagonists 